\title{
The impact of virtual body satisfaction on purchase intentions of a skirt during virtual try-on
}

\author{
Evrim Buyukaslan, Istanbul Bilgi University, Turkey \\ Fatma Baytar, Cornell University, USA \\ Fatma Kalaoglu, Istanbul Technical University, Turkey
}

Keywords: virtual-fit, 3D body scans, fit satisfaction, body satisfaction.

Introduction. In order to increase consumers' garment fit satisfaction and reduce return costs, apparel companies work on integrating virtual try-on technologies to their online shopping activities (Bohnhoff, 2016). Understanding the impact of seeing a virtual-self on body satisfaction is critical because earlier studies show that people tend to have decreased body satisfaction after interacting with their 3D body images (Park, 2018; Ridgway, 2017; Saeidi \& Romeo, 2017). However, these studies did not consider exactly which body parts affected such perceptions. For example, weight and body-build are significant appearance aspects that are related to being thin and being fit, respectively (Pritchard \& Cramblitt, 2014). When people view their 3D body scans during virtual try-on, they may focus on looking at specific areas on the body depending on the garment type to be considered for purchasing during online shopping. As a result, changes in body satisfaction after interacting with one's own virtual body may affect their intentions to purchase garments. Therefore this study is designed to understand the virtual body satisfaction of females between 18 to 35 years old, who comprise the largest online apparel shopper group (Statista, 2019), and their purchase intention of a garment after seeing their body in $3 \mathrm{D}$.

Method. Forty seven females between 18 to 35 ages and medium body size (25-28 inches waist girth, and 35-38 inches hip girth) were recruited at a U.S. university. Each participant was scanned in their underwear by using a Human Solutions Vitus Smart XXL 3D body scanner, and interacted with their 3D scan image on a computer screen for one minute. Participants' body satisfaction levels were measured with a 7-point Likert type body satisfaction scale with twentyone-items before and after seeing their 3D body scans. To examine the impact of virtual body satisfaction on participants' purchase intentions, an online shopping scenario that includes virtual-try on of a circular skirt was tested. Skirt was simulated in an EFI Optitex PDS 2D/3D software, and draped on each participant's body scan avatar. The length and waist measurements of the skirt were 18 inches and 28.5 inches, respectfully. Participants' purchase intentions were

Published under a Creative Commons Attribution License (https://creativecommons.org/licenses/by/4.0/), which permits unrestricted use, distribution, and reproduction in any medium, provided the original work is properly cited.

ITAA Proceedings, \#76 - https: / /itaaonline.org 
measured by showing them the virtual skirt and asking them to what extend they would agree to purchase the skirt. The relationship between participants' virtual body satisfaction and purchase intention was explored by Pearson's correlation test, and the difference between real and virtual body satisfaction was investigated by paired t-test analysis. Findings were analyzed in SPSS v. 25

Results. Principle component analysis with Oblimin rotation was conducted to reduce dimensions of the twenty-one-items body satisfaction scale to find out major body areas first. According to the results five factors with Eigen values higher than 1 were extracted, and these factors explained 72 percent of the total variance. After reducing dimensions of the scale, participants' body satisfactions for each factor were calculated separately. Participants had the highest satisfaction with their upper body parts and lowest satisfaction with the area around their waist when they saw their virtual body models (Table 1).

When the scale items were examined individually, significant differences were found in five areas. Paired t-test analysis between body satisfaction levels of participants for before and after seeing their body scans revealed that subjects' body satisfaction for waist $(\mathrm{t}(47)=-3.73, \mathrm{p}<$ $.05)$, abdomen $(\mathrm{t}(1,47)=-4.16, \mathrm{p}<.05)$, weight $(\mathrm{t}(1,47)=-2.56, \mathrm{p}<.05)$, body-build $(\mathrm{t}(1,47)=-3.85$, $\mathrm{p}<.05)$, and appearance $(\mathrm{t}(1,47)=-2.4, \mathrm{p}<.05)$ dropped significantly after seeing their 3D body scans. There was no significant difference for other body parts.

Table 1. Participants' average virtual body satisfactions based on five areas

\begin{tabular}{cccc}
\hline \hline Body area & N & M & SD \\
\hline Upper body & 45 & 5.53 & 1.04 \\
Stance & 45 & 5.17 & 1.16 \\
Lower body & 45 & 5.10 & 1.18 \\
Around hips & 45 & 4.02 & 1.26 \\
Around waist & 45 & 3.86 & 1.40 \\
\hline
\end{tabular}

The Pearson's correlation test between participants' virtual body satisfactions and their purchase intention for the virtual skirt showed that there were no significant correlation between any of the virtual body satisfactions and participants' purchase intentions.

Conclusion and Implications. In this study, particular changes in virtual body satisfaction was explored in order to see its impact on purchase intention upon online shopping. It was found that participants had the lowest virtual body satisfaction with the waist area. However, no significant correlation was found between participants' purchase intention and virtual body satisfaction according to their virtual try-on. The significant change of body satisfaction for waist, abdomen, permits unrestricted use, distribution, and reproduction in any medium, provided the original work is properly cited.

ITAA Proceedings, \#76 - https://itaaonline.org 
body-build, weight and appearance after seeing the 3D body scan may support that subjects' discrepancy of their self with the ideal beauty norms increase after seeing their body scans. This finding may further be explored by exploring ideal beauty understanding of consumers and their body satisfaction shift for significant body parts.

\section{References}

Bohnhoff, T. (2016). E-commerce: Fashion. Statista Digital Outlook. Retrieved from https://www.statista.com/study/38340/ecommerce-report-fashion/

Park, J. (2018). The effect of virtual avatar experience on body image discrepancy, body satisfaction and weight regulation intention. Cyberpsychology, 12(1).

Pritchard, M., \& Cramblitt, B. (2014). Media influence on drive for thinness and drive for muscularity. Sex Roles, 71(5), 208-218.

Ridgway, J. L. (2017). Before and After Avatar Exposure: The Impact of Body Scanning Technology on Body Satisfaction, Mood, and Appearance Management. Clothing and Textiles Research Journal, 36(2),

Saeidi, E., \& Romeo, L. (2017). Women's Reaction to Whole 3D Body Scanning and its Influence on Body Satisfaction. In International Textile and Apparel Association (ITAA) 2017 Proceedings (pp. 3-5). Florida: International Textile and Apparel Association Inc.

Shin, E., \& Baytar, F. (2013). Apparel fit and size concerns and intentions to use virtual try-on: Impacts of body satisfaction and images of models' bodies. Clothing and Textiles Research Journal, 32(1), 20-33.

Statista. (2019). Share of individuals who purchased clothes and sports goods online in Great Britain in 2018, by age and gender. 